\title{
On images of complete topologized subsemilattices in sequential semitopological semilattices
}

\section{Taras Banakh ${ }^{1,2} \cdot$ Serhii Bardyla ${ }^{3}$}

Received: 5 March 2019 / Accepted: 10 September 2019 / Published online: 26 September 2019

(c) The Author(s) 2019

\begin{abstract}
A topologized semilattice $X$ is called complete if each non-empty chain $C \subset X$ has $\inf C \in \bar{C}$ and $\sup C \in \bar{C}$. We prove that for any continuous homomorphism $h: X \rightarrow Y$ from a complete topologized semilattice $X$ to a sequential Hausdorff semitopological semilattice $Y$ the image $h(X)$ is closed in $Y$.
\end{abstract}

Keywords Sequential space $\cdot$ Complete semitopological semilattice . The tower number

This paper is a continuation of the investigations [1-3] of complete topologized semilattices.

A semilattice is any commutative semigroup of idempotents (an element $x$ of a semigroup is called an idempotent if $x x=x$ ).

A semilattice endowed with a topology is called a topologized semilattice. A topologized semilattice $X$ is called a (semi)topological semilattice if the semigroup operation $X \times X \rightarrow X,(x, y) \mapsto x y$, is (separately) continuous.

Each semilattice carries a natural partial order $\leq$ defined by $x \leq y$ iff $x y=x=y x$. Endowed with this partial order, the semilattice is a poset, i.e., partially ordered set. Many properties of a semilattice can be expressed in the language of this partial order. In particular, a subset $C$ of a semilattice (more generally, poset) $X$ is called a chain if

Communicated by Jimmie D. Lawson.

The second author was supported by the Austrian Science Fund FWF (Grant I 3709-N35).

$凶$ Taras Banakh

t.o.banakh@gmail.com

Serhii Bardyla

sbardyla@yahoo.com

1 Ivan Franko National University of Lviv, Lviv, Ukraine

2 Jan Kochanowski University in Kielce, Kielce, Poland

3 Institute of Mathematics, Kurt Gödel Research Center, Vienna, Austria 
any points $x, y \in C$ are comparable in the sense that $x \leq y$ or $y \leq x$. A poset $X$ is called chain-finite if each chain in $X$ is finite.

In [13] Stepp proved that for any homomorphism $h: X \rightarrow Y$ from a chain-finite semilattice to a Hausdorff topological semilattice $Y$ the image $h(X)$ is closed in $Y$. In [1], the authors improved this result of Stepp proving the following theorem.

Theorem 1 For any homomorphism $h: X \rightarrow Y$ from a chain-finite semilattice to a Hausdorff semitopological semilattice $Y$, the image $h(X)$ is closed in $Y$.

A topological counterpart of the notion of a chain-finite poset is the notion of a complete topologized poset. A topologized poset is a poset $(X, \leq)$ endowed with a topology. A topologized poset $X$ is called complete if each chain $C \subset X$ has inf $C$ and $\sup C$ that belong to the closure $\bar{C}$ of the chain $C$ in $X$.

Complete topologized semilattices were introduced in [1] under the name $k$ complete topologized semilattices. But we prefer to call such topologized semilattice complete (taking into account the fundamental role of complete topologized semilattices in the theory of absolutely closed topologized semilattices, see [1-4,7,11]). In [1] the authors proved the following closedness property of complete topologized semilattices.

Theorem 2 For any continuous homomorphism $h: X \rightarrow Y$ from a complete topologized semilattice $X$ to a Hausdorff topological semilattice $Y$ the image $h(X)$ is closed in $Y$.

Theorems 1 and 2 motivate the following (still) open problem.

Problem 1 Assume that $h: X \rightarrow Y$ is a continuous homomorphism from a complete topologized semilattice $X$ to a Hausdorff semitopological semilattice $Y$. Is $h(X)$ closed in $Y$ ?

The answer to Problem 1 is affirmative if the semitopological semilattice $Y$ is functionally Hausdorff [5] or linearly ordered [10].

In this paper we answer Problem 1 affirmatively under the additional condition that the semitopological semilattice $Y$ is sequential. We recall that a topological space $Y$ is sequential if each sequentially closed subset of $Y$ is closed. A subset $A \subset Y$ is called sequentially closed if $A$ contains the limit points of all sequences $\left\{a_{n}\right\}_{n \in \omega} \subset A$ that converge in $Y$.

The following theorem is the main result of this paper.

Theorem 3 For any continuous homomorphism $h: X \rightarrow Y$ from a complete topologized semilattice $X$ to a sequential Hausdorff semitopological semilattice $Y$ the image $h(X)$ is closed in $Y$.

Theorem 3 will be proved in Sect. 3 after some preliminary work made in Sects. 1 and 2. More precisely, Theorem 3 is an immediate corollary of Corollary 4 treating homomorphisms from complete topologized semilattices to Hausdorff semitopological semilattices of countable tightness. 


\section{Some properties of $\uparrow \omega$-complete topologized semilattices}

In this section we shall prove some properties of complete topologized semilattices, which will be used in the proof of our main results.

First we introduce a parametric version of completeness. Let $\kappa$ be an infinite cardinal. A topologized semilattice $X$ is defined to be

- $\uparrow \kappa$-complete if any non-empty chain $C \subset X$ of cardinality $|C| \leq \kappa$ has sup $C \in \bar{C}$;

- $\downarrow \kappa$-complete if any non-empty chain $C \subset X$ of cardinality $|C| \leq \kappa$ has inf $C \in \bar{C}$;

- $\uparrow \kappa$-complete if $X$ is $\uparrow \kappa$-complete and $\downarrow \kappa$-complete;

- down-complete if $X$ is $\downarrow \kappa$-complete for any cardinal $\kappa$.

It is clear that a topologized semilattice $X$ is complete if and only if $X$ is $\uparrow \kappa$-complete for every cardinal $\kappa$ if and only if $X$ is $\uparrow \kappa$-complete for the cardinal $\kappa=|X|$.

A subset $D$ of a poset $(X, \leq)$ is called up-directed (resp. down-directed) if for any elements $x, y \in D$ there exists an element $z \in D$ such that $x \leq z$ and $y \leq z$ (resp. $z \leq x$ and $z \leq y$ ). It is clear that each chain is both up-directed and down-directed.

Lemma 1 If a topologized semilattice $X$ is $\uparrow \omega$-complete, then any non-empty countable up-directed subset $D \subset X$ has $\sup D \in \bar{D}$.

Proof Assume that the topologized semilattice $X$ is $\uparrow \omega$-complete and take any nonempty countable directed subset $D=\left\{x_{n}\right\}_{n \in \omega}$ in $X$. Put $y_{0}:=x_{0}$ and for every $n \in \mathbb{N}$ choose an element $y_{n} \in D$ such that $y_{n} \geq x_{n}$ and $y_{n} \geq y_{n-1}$ (such an element $y_{n}$ exists as $D$ is directed).

By the $\uparrow \omega$-completeness, the chain $C:=\left\{y_{n}\right\}_{n \in \omega}$ has sup $C \in \bar{C} \subset \bar{D}$. We claim that $\sup C$ is the smallest upper bound of the set $D$. Indeed, for any $n \in \omega$ we get $x_{n} \leq y_{n} \leq \sup D$ and hence $\sup C$ is an upper bound for the set $D$. On the other hand, each upper bound $b$ for $D$ is an upper bound for $C$ and hence $\sup C \leq b$. Therefore $\sup D=\sup C \in \bar{C} \subset \bar{D}$.

Lemma 2 If a topologized semilattice $X$ is $\downarrow \omega$-complete, then each non-empty countable subset $A \subset X$ has inf $A \in \bar{S}$ where $S$ is the semilattice generated by $A$ in $X$.

Proof Let $A=\left\{x_{n}\right\}_{n \in \omega}$ be a countable set in $X$. By the $\downarrow \omega$-completeness of $X$, the chain $C:=\left\{x_{0} \cdots x_{n}\right\}_{n \in \omega} \subset S$ has inf $C \in \bar{C} \subset \bar{S}$. Taking into account that $\inf A=\inf C$, we conclude that inf $A=\inf C \in \bar{S}$.

Let $X$ be an $\uparrow \omega$-complete topologized semilattice, $A$ be a non-empty countable set in $X$ and $S$ be a subsemilattice of $X$, generated by $A$. Let $[A]^{<\omega}$ be the family of all finite subsets of $A$. Lemma 2 implies that for each $F \in[A]^{<\omega}$ the set $A \backslash F$ has $\inf (A \backslash F) \in \bar{S}$. By Lemma 1 , the directed set $D:=\left\{\inf (A \backslash F): F \in[A]^{<\omega}\right\}$ has $\sup D \in \bar{D} \subset \bar{S}$. The element

$$
\inf ^{*} A:=\sup _{F \in[A]<\omega} \inf (A \backslash F)=\sup D \in \bar{S}
$$

will be called the essential infimum of the set $A$ in $X$. 
An important property of the essential infimum is that inf* $B \leq$ inf* $^{*}$ for any countable subset $A \subset^{*} B$ in $X$. Here the symbol $A \subset^{*} B$ means that the complement $A \backslash B$ is finite, so $A$ is almost included into $B$.

We shall say that an infinite subset $A$ of a topological space $X$ converges to a point $x \in X$ if each neighborhood $O_{x} \subset X$ of $x$ contains all but finitely many points of the set $A$ (which means that $A \subset^{*} O_{x}$ ). If an infinite set $A$ converges to a point $x$, then any infinite subset $B \subset A$ also converges to $x$.

We are going to show that for any $\uparrow \omega$-complete Hausdorff topologized semilattice $X$ containing no strictly increasing transfinite sequences of length $\mathfrak{t}$, any countable subset $A \subset X$ that converges to a point $x$ contains an infinite subset $B \subset A$ such that

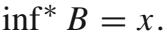

The cardinal $\mathfrak{t}$ (called the tower number in [14]) is defined as the smallest cardinal $\kappa$ for which there exists a transfinite sequence $\left(T_{\alpha}\right)_{\alpha \in \kappa}$ of infinite subsets of $\omega$ having the following two properties:

(1) $T_{\beta} \subset^{*} T_{\alpha}$ for all $\alpha<\beta<\kappa$;

(2) for any infinite set $I \subset \omega$ there exists $\alpha \in \kappa$ such that $I \not \subset^{*} T_{\alpha}$.

It is known $[14,15]$ that $\omega_{1} \leq \mathfrak{t} \leq \mathfrak{c}$, and $\mathfrak{t}=\mathfrak{c}$ under Martin's Axiom. By a recent breakthrough result of Malliaris and Shelah [12], $\mathfrak{t}$ is equal to the pseudointersection number $\mathfrak{p}$, defined as the smallest cardinality of a non-empty family $\mathcal{A}$ of subsets of $\omega$ such that for any finite subfamily $\mathcal{F} \subset \mathcal{A}$ the intersection $\cap \mathcal{F}$ is infinite but for any infinite set $I \subset \omega$ there exists $A \in \mathcal{A}$ such that $I \not \not^{*} A$. More information on cardinals $\mathfrak{p}, \mathfrak{t}$ and other cardinal characteristics of the continuum can be found in the surveys $[8,14,15]$. We identify cardinals with the smallest ordinals of a given cardinality.

Let $\kappa$ be an ordinal. A transfinite sequence $\left(x_{\alpha}\right)_{\alpha \in \kappa}$ of points of a partially ordered set $X$ is called strictly increasing if $x_{\alpha}<x_{\beta}$ for any ordinals $\alpha<\beta$ in $\kappa$.

Lemma 3 Let $Y$ be a Hausdorff semitopological semilattice and $X$ be an $\uparrow \omega$-complete subsemilattice of $Y$ containing no strictly increasing transfinite sequences of length $\mathrm{t}$. Then each infinite set $A \subset X$ that converges to a point $y \in Y$ contains an infinite subset $B \subset A$ such that inf* $B=y$.

Proof Fix an infinite set $A \subset X$ that converges to a point $y \in Y$. To derive a contradiction, assume that inf* $B \neq y$ for any infinite subset $B \subset A$.

Claim 1 For any infinite subset $B \subset A$ there exists an infinite subset $C \subset B$ such that

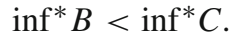

Proof By our assumption, inf* $B \neq y$. By the Hausdorff property of $Y$, there exists an open neighborhood $U \subset Y$ of $y$ such that inf* $B \notin \bar{U}$.

Inductively we shall construct a sequence of pairwise distinct points $\left\{x_{n}\right\}_{n \in \omega}$ in $B$ such that for every $k \leq n$ the product $y_{k, n}:=x_{k} \cdots x_{n} y$ is contained in $U$.

To start the inductive construction, find a neighborhood $V_{0} \subset U$ of $y$ such that $V_{0} y \subset U$ and choose any point $x_{0} \in V_{0} \cap B$ (such a point exists as $B \subset A$ converges to $y$ ). It follows that $x_{0} y \in V_{0} y \subset U$.

Assume that for some $n \in \omega$ points $x_{0}, \ldots, x_{n} \in B$ are chosen so that $y_{k, n}:=$ $x_{k} \cdots x_{n} y \in U$ for every $k \leq n$. For every $k \leq n$ choose a neighborhood $V_{k, n} \subset U$ of 
$y$ such that $y_{k, n} V_{k, n} y \subset U$ (such neighborhood exists since $y_{k, n} y y=y_{k, n} \in U$ and $Y$ is a semitopological semilattice). Consider the neighborhood $V_{n+1}:=\bigcap_{k \leq n} V_{k, n}$ of $y$ and choose any point $x_{n+1} \in V_{n+1} \cap B \backslash\left\{x_{0}, \ldots, x_{n}\right\}$. For every $k \leq n$ the choice of the neighborhood $V_{k, n}$ guarantees that $y_{k, n+1}:=x_{k} \cdots x_{n} x_{n+1} y \in U$. This completes the inductive step.

After completing the inductive construction, consider the set $C=\left\{x_{k}\right\}_{k \in \omega} \subset B$. We claim that inf* $C=y \cdot$ inf* $^{*} \in \bar{U}$. For every $k \in \omega$ consider the set $C_{k}=$ $\left\{x_{n}\right\}_{n \geq k} \subset C$ and let $S_{k}$ be the subsemilattice generated by $C_{k}$ in $X$. By the $\downarrow \omega$ completeness of $X$ and Lemma 2, the set $C_{k}$ has inf $C_{k}=\inf S_{k} \in \bar{S}_{k}$. In fact, $\inf C_{k}=\inf S_{k}=\inf \left\{x_{k} \cdots x_{n}: n \geq k\right\} \in \operatorname{cl}_{X}\left(\left\{x_{k} \cdots x_{n}: n \geq k\right\}\right)$. Observe that $\inf C_{k} \leq x_{n}$ for all $n \geq k$. Consequently, $y \in \operatorname{cl}_{Y}\left(\left\{x_{n}\right\}_{n \geq k}\right) \subset \uparrow \inf C_{k}$ and hence $y \cdot \inf C_{k}=\inf C_{k}$.

The continuity of the shift $s_{y}: Y \rightarrow Y, s_{y}: z \mapsto z y$, guarantees that inf $C_{k}=$ $y \cdot \inf C_{k} \in y \cdot \operatorname{cl}_{X}\left(\left\{x_{k} \cdots x_{n}: n \geq k\right\}\right) \subset \operatorname{cl}_{Y}\left(\left\{x_{k} \cdots x_{n} y: n \geq k\right\}\right) \subset \bar{U}$.

By the $\uparrow \omega$-completeness of $X$, we have inf* $C=\sup _{k \in \omega} \inf C_{k} \in \operatorname{cl}_{X}\left(\left\{\inf C_{k}\right.\right.$ : $k \in \omega\}) \subset \bar{U}$ and hence inf $^{*} C \neq$ inf* $^{*}$ as inf* $B \notin \bar{U}$. Taking into account that

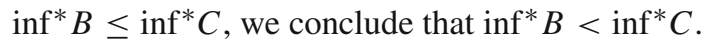

Choose any countable infinite subset $A_{0} \subset A$. Claim 1 (on successor steps) and the definition of the cardinal $\mathfrak{t}$ (on limit steps) help us to construct a transfinite sequence $\left(A_{\alpha}\right)_{\alpha<\mathfrak{t}}$ of infinite subsets of $A_{0}$ such that for any $\alpha<\mathfrak{t}$ the following conditions are satisfied:

(1) $A_{\alpha+1} \subset A_{\alpha}$ and inf* $A_{\alpha}<$ inf* $^{*} A_{\alpha+1}$.

(2) $A_{\alpha} \subset^{*} A_{\gamma}$ for all $\gamma<\alpha$.

Then $\left(\text { inf }^{*} A_{\alpha}\right)_{\alpha \in \mathfrak{t}}$ is a strictly increasing transfinite sequence of length $\mathfrak{t}$ in $X$, whose existence is forbidden by our assumption. This contradiction completes the proof of the lemma.

We recall that a topological space $X$ is defined to have countable tightness if for any set $A \subset X$ and point $a \in \bar{A}$, there exists a countable set $B \subset A$ such that $a \in \bar{B}$. It is known $[9,1.7 .3 \mathrm{c}]$ that each subspace of a sequential space has countable tightness.

Lemma 4 A topologized semilattice $X$ contains no strictly increasing transfinite sequences of length $\omega_{1}$ if $X$ is $\uparrow \omega_{1}$-complete, the space $X$ has countable tightness, and for every $x \in X$ the lower set $\downarrow x$ is closed in $X$.

Proof To derive a contradiction, assume that $X$ contains a strictly increasing transfinite sequence $\left(x_{\alpha}\right)_{\alpha \in \omega_{1}}$. By the $\uparrow \omega_{1}$-completeness of $X$ the chain $C=\left\{x_{\alpha}: \alpha \in \omega_{1}\right\}$ has $c:=\sup C \in \bar{C}$. Since $\left(x_{\alpha}\right)_{\alpha \in \omega_{1}}$ is strictly increasing, $c \notin C$. By the countable tightness of $X$, there exists a countable set $B \subset C$ such that $c \in \bar{B}$. By the countability of $B$, there exists a countable ordinal $\beta$ such that $B \subset\left\{x_{\alpha}: \alpha<\beta\right\}$. Then $c \in \bar{B} \subset$ $\overline{\downarrow x_{\beta}}=\downarrow x_{\beta}$ and hence $c \leq x_{\beta}<x_{\beta+1} \leq c$, which is a desired contradiction.

\section{The closedness of complete subsemilattices}

In this section we search for conditions of (sequential) closedness of a $\uparrow \omega$-complete semilattice $X$ in a Hausdorff semitopological semilattice $Y$. 
Theorem 4 Let $Y$ be a Hausdorff semitopological semilattice and $X$ be $a \uparrow \omega$-complete subsemilattice of $Y$. If $X$ contains no strictly increasing transfinite sequences of length $\mathfrak{t}$, then $X$ is sequentially closed in $Y$ and the partial order $\{(x, y) \in X \times X: x y=x\}$ of $X$ is sequentially closed in $Y \times Y$.

Proof Assuming that $X$ is not sequentially closed in $Y$, we can find a sequence $\left(x_{n}\right)_{n \in \omega}$ of pairwise distinct points of $X$ that converges to a point $y \in Y \backslash X$. It follows that the infinite set $A=\left\{x_{n}\right\}_{n \in \omega} \subset X$ converges to $y$. By Lemma 3, the set $A$ contains an infinite subset $B \subset A$ such that $y=\inf ^{*} B \in X$. But this contradicts the choice of $y$.

To show that the partial order $P=\{(x, y) \in X \times X: x y=x\}$ of $X$ is sequentially closed in $Y \times Y$, fix any sequence $\left\{\left(x_{n}, y_{n}\right)\right\}_{n \in \omega} \subset P$ that converges to a pair $(x, y) \in$ $Y \times Y$. We should prove that $(x, y) \in P$. Since $X$ is sequentially closed in $Y$, the limits $x, y$ of the sequences $\left(x_{n}\right)_{n \in \omega}$ and $\left(y_{n}\right)_{n \in \omega}$ belong to $X$. The separate continuity of the semigoup operation on the Hausdorff space $X$ implies that the sets $\uparrow x:=\{z \in$ $Y: z x=x\}$ and $\downarrow y:=\{z \in Y: z y=z\}$ are closed in $Y$.

If the set $\left\{x_{n}\right\}_{n \in \omega}$ is finite, then the convergence $x=\lim _{n \rightarrow \infty} x_{n}$ implies that the set $I:=\left\{n \in \omega: x_{n}=x\right\}$ is infinite and then $y \in \operatorname{cl}_{Y}\left(\left\{y_{n}\right\}_{n \in I}\right) \subset \uparrow x\left(\right.$ as $x=x_{n} \leq y_{n}$ for all $n \in I)$ and hence $(x, y) \in P$. So, we can assume that the set $\left\{x_{n}\right\}_{n \in \omega}$ is infinite. Applying Lemma 3, we can find an infinite set $\Omega \subset \omega$ such that $x=\inf ^{*}\left\{x_{n}\right\}_{n \in \Omega}$.

If the set $\left\{y_{n}\right\}_{n \in \Omega}$ is finite, then the convergence $y=\lim _{n \rightarrow \infty} y_{n}$ implies that the set $J:=\left\{n \in \Omega: y_{n}=y\right\}$ is infinite and then $x \in \operatorname{cl}_{X}\left(\left\{x_{n}\right\}_{n \in J}\right) \subset \downarrow y$ and $(x, y) \in P$.

So we can assume that the set $\left\{y_{n}\right\}_{n \in \Omega}$ is infinite. Applying Lemma 3, we can find an infinite set $\Lambda \subset \Omega$ such that $y=\inf ^{*}\left\{y_{n}\right\}_{n \in \Lambda}$. Taking into account that $x_{n} \leq y_{n}$ for all $n \in \omega$, we see that $x=\inf ^{*}\left\{x_{n}\right\}_{n \in \Omega} \leq \inf ^{*}\left\{x_{n}\right\}_{n \in \Lambda} \leq \inf ^{*}\left\{y_{n}\right\}_{n \in \Lambda}=y$ and hence $(x, y) \in P$.

Theorem 4 and Lemma 4 imply

Corollary 1 Let $X$ be a subsemilattice of a Hausdorff semitopological semilattice $Y$ such that $X$ is $\downarrow \omega$-complete and $\uparrow \omega_{1}$-complete. If $X$ has countable tightness, then $X$ is sequentially closed in $Y$ and the partial order $\{(x, y) \in X \times X: x y=x\}$ of $X$ is sequentially closed in $Y \times Y$.

Taking into account that subspaces of sequential spaces have countable tightness $[9,1.7 .3 \mathrm{c}]$, we can see that Corollary 1 implies another corollary.

Corollary 2 Let X be a subsemilattice of a sequential Hausdorff semitopological semilattice $Y$. If $X$ is $\downarrow \omega$-complete and $\uparrow \omega_{1}$-complete, then $X$ is closed in $Y$ and the partial order $\{(x, y) \in X \times X: x y=x\}$ of $X$ is sequentially closed in $Y \times Y$.

Remark 1 The $\downarrow \omega$-completeness of $X$ is essential in Corollaries 1 and 2: by [6], there exists a metrizable semitopological semilattice $X$ whose partial order is not closed in $X \times X$, and for every $x \in X$ the upper set $\uparrow x$ is finite.

\section{The closedness of images of complete semilattices}

In this section we apply the results of the preceding section to establish the (sequential) closedness of images of complete semilattices under continuous homomorphisms. 
Lemma 5 Let $h: X \rightarrow Y$ be a surjective continuous homomorphism from a downcomplete topologized semilattice $X$ to a Hausdorff semitopological semilattice $Y$. Let $\kappa$ be a cardinal.

(1) The topologized semilattice $Y$ is down-complete.

(2) If $X$ is $\uparrow \kappa$-complete, then $Y$ is $\uparrow \kappa$-complete.

(3) If $X$ contains no strictly increasing transfinite sequences of length $\kappa$, then $Y$ contains no strictly increasing transfinite sequences of length $\kappa$.

Proof For every $y \in Y$ consider the closed subsemilattice $S_{y}:=h^{-1}(\uparrow y)$ in $X$. Let $M_{y}$ be a maximal chain in $S_{y}$. By the down-completeness of $X$, the chain $M_{y}$ has $\inf M_{y} \in \bar{M}_{y} \subset \bar{S}_{y}=S_{y}$. We claim that $s_{y}:=\inf M_{y}$ is the smallest element of $S_{y}$. In the opposite case, there would exist an element $x \in S_{y}$ such that $s_{y} \not \subset x$ and hence $x s_{y}<s_{y}$. Then $\left\{x s_{y}\right\} \cup M_{y}$ is a chain in $S_{y}$, properly containing the maximal chain $M_{y}$, which is a desired contradiction showing that $s_{y}$ is the smallest element min $S_{y}$ of the semilattice $S_{y}$. It follows from $h\left(S_{y}\right)=\uparrow y$ that $h\left(s_{y}\right) \in h\left(S_{y}\right)=\uparrow y$ and hence $y \leq h\left(s_{y}\right)$. On the other hand, for any $x \in h^{-1}(y)$ we get $s_{y} \leq x$ and hence $h\left(s_{y}\right) \leq h(x)=y$ and finally $h\left(s_{y}\right)=y$. It is clear that for any $x \leq y$ in $C$, we get $S_{x} \subset S_{y}$ and hence $\min S_{x} \leq \min S_{y}$.

1. To prove that $Y=h(X)$ is down-complete, we should show that any nonempty chain $C \subset Y$ has $\inf C \in \bar{C}$. It follows that for any $x \leq y$ in $C$, we get $\min S_{x} \leq \min S_{y}$, which means that $D:=\left\{\min S_{x}\right\}_{x \in C}$ is a chain in $X$. By the down-completeness of $X$, the chain $D$ has inf $D \in \bar{D}$.

The continuity of the homomorphism $h$ ensures that $h($ inf $D) \in h(\bar{D}) \subset \overline{h(D)}=$ $\bar{C}$. It remains to check that $h(\inf D)=\inf C$. Taking into account that $h$ is a semilattice homomorphism, we can show that $h(\inf D)$ is a lower bound of the set $C=h(D)$ in $Y=h(X)$. For any other lower bound $b \in h(X)$ of $C$, we see that $C \subset \uparrow b$, $D \subset h^{-1}(\uparrow b)=S_{b}$ and hence $\min S_{b} \leq \inf D$, which implies that $b=h\left(\min S_{b}\right) \leq$ $h(\inf D)$. So, inf $C=h(\inf D) \in \bar{C}$.

2. Assuming that $X$ is $\uparrow \kappa$-complete, we shall check that the topologized semilattice $Y$ is $\uparrow \kappa$-complete. Given any non-empty chain $C \subset Y$ of cardinality $|C| \leq \kappa$, we should show that $C$ has $\sup C \in \bar{C}$. It is clear that for any $x \leq y$ in $C$, we get $\min S_{x} \leq \min S_{x}$, which means that $D:=\left\{\min S_{x}\right\}_{x \in C}$ is a chain in $X$ of cardinality $|D| \leq|C| \leq \kappa$. Since $X$ is $\uparrow \kappa$-complete, the chain $D$ has $\sup D \in \bar{D}$.

The continuity of the homomorphism $h$ ensures that $h(\sup D) \in h(\bar{D}) \subset \overline{h(D)}=$ $\bar{C}$. It remains to check that $h(\sup D)=\sup C$. Taking into account that $h$ is a semilattice homomorphism, we can show that $h(\sup D)$ is an upper bound for the set $C=h(D)$ in $Y=h(X)$. For any other upper bound $b \in h(X)$ of $b$, we see that $C \subset \downarrow b, D \subset$ $\downarrow \min S_{b}$ and hence $\sup D \leq \min S_{b}$, which implies that $h(\sup D) \leq h\left(\min S_{b}\right)=b$. So, $\sup C=h(\sup D) \in \bar{C}$.

3. Assuming that $\left(y_{\alpha}\right)_{\alpha \in \kappa}$ is a strictly increasing transfinite sequence of length $\kappa$ in $Y$, we can see that ( $\left.\min S_{y_{\alpha}}\right)_{\alpha \in \kappa}$ is a strictly increasing transfinite sequence of length $\kappa$ in $X$.

Lemma 5 and Theorem 4 imply: 
Corollary 3 Let $X$ be a down-complete $\uparrow \omega$-complete topologized semilattice containing no strictly increasing transfinite sequences of length $\mathfrak{t}$. For any continuous homomorphism $h: X \rightarrow Y$ to a Hausdorff semitopological semilattice $Y$, the image $h(X)$ is sequentially closed in $Y$.

\section{Lemma 5 and Corollary 1 imply:}

Theorem 5 Let $X$ be a countably tight down-complete $\uparrow \omega_{1}$-complete topologized semilattice. For any continuous homomorphism $h: X \rightarrow Y$ to a Hausdorff semitopological semilattice $Y$, the image $h(X)$ is sequentially closed in $Y$.

Corollary 4 For any continuous homomorphism $h: X \rightarrow Y$ from a down-complete $\uparrow \omega_{1}$-complete topologized semilattice $X$ to a Hausdorff semitopological semilattice $Y$ of countable tightness, the image $h(X)$ is sequentially closed in $Y$.

Since sequential spaces have countable tightness [9, 1.7.13(c)], Corollary 4 implies Theorem 3 announced in the introduction.

Open Access This article is licensed under a Creative Commons Attribution 4.0 International License, which permits use, sharing, adaptation, distribution and reproduction in any medium or format, as long as you give appropriate credit to the original author(s) and the source, provide a link to the Creative Commons licence, and indicate if changes were made. The images or other third party material in this article are included in the article's Creative Commons licence, unless indicated otherwise in a credit line to the material. If material is not included in the article's Creative Commons licence and your intended use is not permitted by statutory regulation or exceeds the permitted use, you will need to obtain permission directly from the copyright holder. To view a copy of this licence, visit http://creativecommons.org/licenses/by/4.0/.

\section{References}

1. Banakh, T., Bardyla, S.: Characterizing chain-finite and chain-compact topological semilattices. Semigroup Forum 98(2), 234-250 (2019)

2. Banakh, T., Bardyla, S.: Completeness and absolute $H$-closedness of topological semilattices. Topol. Appl. 260, 189-202 (2019)

3. Banakh, T., Bardyla, S.: The interplay between weak topologies on topological semilattices. Topol. Appl. Topol. Appl. 259, 134-154 (2019)

4. Banakh, T., Bardyla, S.: Complete topologized posets and semilattices. Preprint (arXiv:1806.02869)

5. Banakh, T., Bardyla, S., Ravsky, A.: The closedness of complete subsemilattices in functionally Hausdorff semitopological semilattices. Topol. Appl. 267, 106874 (2019)

6. Banakh, T., Bardyla, S., Ravsky, A.: A metrizable semitopological semilattice with non-closed partial order. Preprint (arXiv:1902.08760)

7. Bardyla, S., Gutik, O.: On $\mathcal{H}$-complete topological semilattices. Mat. Stud. 38(2), 118-123 (2012)

8. Blass, A.: Combinatorial cardinal characteristics of the continuum. In: Foreman, M., Kanamori, A. (eds.) Handbook of Set Theory, pp. 395-489. Springer, Dordrecht (2010)

9. Engelking, R.: General Topology. Heldermann, Berlin (1989)

10. Gutik, O.V.: On closures in semitopological inverse semigroups with continuous inversion. Algebra Discrete Math. 18(1), 59-85 (2014)

11. Gutik, O., Repovš, D.: On linearly ordered $H$-closed topological semilattices. Semigroup Forum 77(3), 474-481 (2008)

12. Malliaris, M., Shelah, S.: General topology meets model theory, on $\mathfrak{p}$ and $t$. Proc. Natl. Acad. Sci. USA 110(33), 13300-13305 (2013)

13. Stepp, J.W.: Algebraic maximal semilattices. Pac. J. Math. 58(1), 243-248 (1975) 
14. van Douwen, E.K.: The integers and topology. In: Kunen, K., Vaughan, J. (eds.) Handbook of SetTheoretic Topology, pp. 111-167. North-Holland, Amsterdam (1984)

15. Vaughan, J.: Small uncountable cardinals and topology. In: van Mill, J., Reed, G.M. (eds.) Open Problems in Topology, pp. 195-218. North-Holland, Amsterdam (1990)

Publisher's Note Springer Nature remains neutral with regard to jurisdictional claims in published maps and institutional affiliations. 